\title{
Contribution of somesthetic cues to the perception of body orientation and subjective visual vertical
}

\author{
MARION TROUSSELARD and CORINNE CIAN \\ Centre de Recherches du Service de Santé des Armées, La Tronche, France \\ VINCENT NOUGIER \\ Université Joseph Fourier, Grenoble, France \\ and \\ SIMON PLA and CHRISTIAN RAPHEL \\ Centre de Recherches du Service de Santé des Armées, La Tronche, France
}

\begin{abstract}
Without relevant visual cues, the subjective visual vertical (SVV) is biased in roll-tilted subjects toward the body axis (Aubert or A-effect). This study focused on the role of the somatosensory system with respect to the SVV and on whether somesthetic cues act through the estimated body tilt. The body cast technology was used to obtain a diffuse tactile stimulation. An increased A-effect was expected because of a greater underestimation of the body position in the body cast. Sixteen subjects placed in a tilt chair were rolled sideways from $0^{\circ}$ to $105^{\circ}$. They were asked to verbally indicate their subjective body position and then to adjust a luminous line to the vertical under strapped and body cast conditions. Results showed a greater A-effect $(p<.001)$ but an overestimation of the body orientation $(p<$ .01 ) in the body cast condition for the higher tilt values (beyond $60^{\circ}$ ). Since the otolith organs produced the same gravity response in both conditions, errors were due to a change in somesthetic cues. Visual and postural errors were not directly related (no correlation). However, the angular distance between the apparent body position and the SVV remained stable, suggesting that the change in somatosensory pattern inputs has a similar impact on the cognitive processes involved in assessing the perception of external space and the sense of self-position.
\end{abstract}

In an otherwise dark environment, a person in an upright position can align the orientation of a visual line with the direction of gravity (subjective visual vertical, SVV) very accurately (Witkin \& Asch, 1948). However, two types of systematic deviations between the perceived and the gravitational vertical can be observed when the person is tilted sideways. For moderate tilts (smaller than $60^{\circ}$ ), the final setting of the visual line deviates from the true vertical contralaterally to the body tilt (Müller or E-effect). For greater tilts, the SVV deviates from the true vertical in the same direction as the body tilt (Aubert or A-effect). The dissimilarities observed between A-effects and E-effects are usually explained by a difference in the perceived body tilt. It is often argued that small angles of body tilt are associated with an overestimation of one's own body position (E-effect) and large angles of body tilt are associated with an underestimation (A-effect; Day \& Wade, 1969; Howard \& Templeton, 1966; Parker, Poston, \& Gulledge, 1983). Thereby, it is assumed that the SVV

Correspondence concerning this article should be addressed to M. Trousselard, Centre de Recherches du Service de Santé des Armées, 38702 La Tronche, Cedex, France (e-mail: mtrousselard@ crssa.net). and the perception of body position are based on the same reference for the physical vertical (Van Beuzekom \& Van Gisbergen, 2000). Regarding the estimation of body tilt, results are quite inconsistent. Higashiyama and Koga (1998) recorded some fairly accurate estimates up to a $45^{\circ}$ roll tilt. At higher roll tilts, the values were overestimated. On the other hand, Van Beuzekom and Van Gisbergen (2000) obtained verbal underestimations of the body roll tilt at angles between $0^{\circ}$ and $180^{\circ}$. For tilt angles exceeding $60^{\circ}$, they also observed a correlation between errors made in body tilt estimates and errors produced in the subjective vertical task. Conversely, Mast and Jarchow (1996) recorded the subjective visual horizontal when subjects had the feeling that they were lying horizontally on the side. They found a paradoxical phenomenon. Although the subjects felt perfectly horizontal, they reported that the luminous line parallel to their spinal axis did not appear horizontal.

The A-effect is considered to be a consequence of the changes in the vestibular (namely, the otolith) and somatosensory inputs secondary to a body tilt in the dark (Bronstein, 1999). It has been shown that the ability to position a luminous bar on the gravitational vertical in an otherwise darkened room varies proportionally to the 
shear force in the utricle (Schöne \& Wade, 1971). Therefore, the SVV is shown to depend in part on the otolith function (Schöne, 1964), and the decrease in precision (A-effect) is related to the decrease in utricle sensitivity as the body tilt increases (Mittelstaedt, 1983; Schöne, 1975). On the other hand, when a subject is tilted, the somatosensory information from skin receptors also changes. Because this information reaches conscious perception, it has been persistently argued that it is also used as an important reference for the control and perception of posture. This sensory system acts through the muscular and articulatory endocaptors, the cutaneous exocaptors, and interoception - that is, afferent signals from the visceral organs (Mittelstaedt, 1992, 1997). However, the weighting of the sensory systems implied in spatial orientation remains unclear. It may be assumed that the somatosensory system is likely to play an increasing role in the determination of body orientation with gradually greater body tilts (Schöne, 1975), whereas the otolith organs become less sensitive.

Concerning the issue of somesthetic contribution, the difficulties involved in investigating the somatosensory system should be stressed, since any experimental option will have its disadvantage. In research carried out under immersion, the proprioceptive, tactile, and interoceptive inputs are artificially disturbed. Indeed, gravity loads on the joints are reduced, and pressure is equalized over the whole body. According to certain data, SVV adjustment errors become greater under immersion versus normal conditions at tilt values higher than $40^{\circ}$ (Schöne, 1964; Wade, 1973). Other research has identified only minor effects (Jarchow \& Mast, 1999; Lechner-Steinleitner \& Schöne, 1980). Where body tilt estimations are concerned, tilted divers could not correctly assess their body position (Brown, 1961; Nelson, 1968). Methodological problems typical of such experiments-namely, equipment-related respiratory difficulties, differences in the floatability of various body parts-may account for the lack of consensus. Also, in experiments conducted under microgravity, the gravity force, which interacts with the vestibular and somesthetic systems, is canceled. Moreover, in the absence of visual cues, the subject is left without spatial reference, and the vertical reference is transferred to the body axis (Raphan \& Sturm, 1992). One can easily understand how difficult it would be to conduct experiments aimed at identifying the somesthetic phenomena involved in spatial orientation. Case studies on subjects with no proprioception are scarce, and they are often biased: Etiology is variable, and lesions are rarely "pure," thus making it difficult to interpret and draw generalizations from the results. Several studies showed that patients with no somatosensory function below the neck (Yardley, 1990) and patients with hemisensory loss lying on their anesthetized side (Anastasopoulos, Bronstein, Haslwanter, Fetter, \& Dichgans, 1999) did not exhibit the expected A-effect when tilted in the way that normally induced an A-effect. They indicated that this tilt-mediated effect seems to be of somatosensory origin (Bronstein, 1999).
In other studies, diffuse tactile stimulations were produced using a padded structure in order to modify tactile orientation information over the whole body (Nemire \& Cohen, 1993; Nyborg, 1971). These studies showed that the new somesthetic pattern reduces accuracy in exocentered and egocentered spatial orientation tasks. More recently, Bringoux, Nougier, Barraud, Marin, and Raphel (in press), using a body cast technology to create a more diffuse and isotropic pattern of pressure stimulation, found an increase in body underestimation when the body was tilted in the pitch dimension. Thus, there is convincing experimental evidence that the somatosensory system plays an important role in spatial orientation.

The aim of this experiment was to show whether the visual vertical is determined directly by the felt orientation of the body. To determine how estimates of body tilt can account for the SVV, we evaluated the verbal estimates of body tilt and the SVV settings while the somatosensory cues were attenuated by means of a body cast. With regard to the results observed by Bringoux et al. (in press), our main hypothesis postulated a greater underestimation of body position for larger roll tilts when the relevant somatosensory inputs were disturbed by the body cast. Consequently, an increased A-effect was expected.

\section{METHOD}

\section{Subjects}

Sixteen naive subjects, who were all volunteers ( 5 females and 11 males; mean age, 28 years), took part in the experiment. All the subjects had normal or corrected-to-normal vision and were free of any apparent vestibular or somesthetic abnormalities. In conformity with the Helsinki Convention, which controls and regulates human experimentation, informed consent was obtained from all the subjects.

\section{Apparatus}

The subject was placed in a chair on an apparatus capable of rotating to any angle in the frontal plane with a $3 \% \mathrm{sec}$ constant velocity and a starting acceleration and a final deceleration of $0.01 \% / \mathrm{sec}^{2}$ (Figure 1). The pivot on which the body rotated was approximately at midpoint between the eyes. The subject was restrained in the chair with his or her head aligned with the body by means of straps (control condition) or the body cast. In the strapped condition, the subject was immobilized by means of five large straps fixed at the levels of the breast, abdomen, hips, knees, and ankles. The subject's head was held by a helmet. A stiff side support surface provided ample cutaneous input. In the body cast condition, the subject was completely immobilized by means of four plastic envelopes filled with micro-marbles and connected to a depressurization device. The surface of the plastic envelopes was very thin in order to ensure the closest possible contact with the subject's body. The first envelope partly covered the subject's trunk and shoulders. The second envelope surrounded the hips. The third envelope specifically immobilized the head, except for the face. The last one was wrapped around the knees and the feet. In roll tilt, the homogenization of pressure on the gluteal side, head, and support surface-for any body orientation - reduces the asymmetry of deep pressure.

The subject faced a monitored 32-cm-long and 1.5-cm-wide luminous rod secured to the platform at a $55-\mathrm{cm}$ distance. The rod was a linear array of equally spaced LEDs covered by a translucent plastic sheet; the luminance of the target was $0.3 \mathrm{~cd} / \mathrm{m}^{2}$ (Spectrascan 650). The adjustable line was rotated on the subject's frontal 

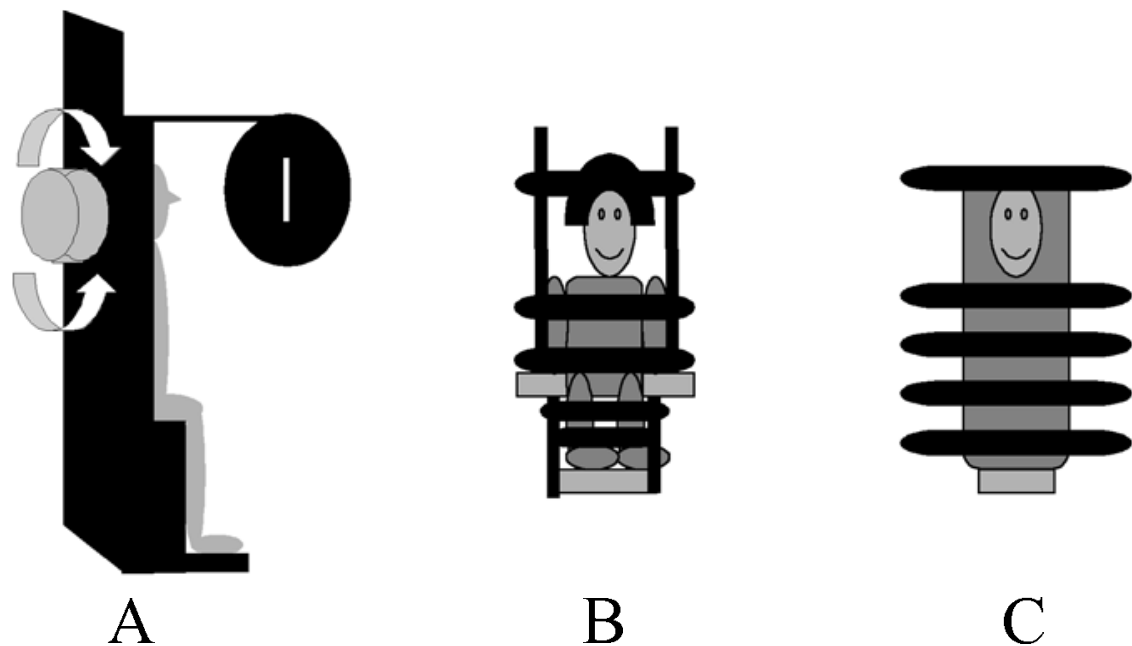

Figure 1. Apparatus (A) and immobilization in the strapped condition (B) and the body cast condition $(C)$. The platform could produce any desired body roll tilt. A position and velocity interlocking system provided a 1/200th accuracy. The luminous rod tilted in the frontal plane, with a velocity interlocking system allowing for fast and accurate adjustments. The subject's hands and the joystick are not described in the figure.

plane, approximately on the midpoint of the interocular axis at a velocity of $5 \% \mathrm{sec}$, by means of a joystick held in the subject's hands lying on his or her thighs. The platform position and the settings of the luminous rod were controlled and recorded at a $0.01^{\circ}$ accuracy, using a digital position encoder.

\section{Procedure}

The subject, placed in an otherwise dark room, was instructed to keep his or her eyes closed at all times, except during rod adjustment. In a given trial, the chair was rotated and was stopped at the preset position. After a delay of $30 \mathrm{sec}$, to eliminate possible transitory effects due to the postrotary deflection of the cupula (Howard, 1986), the subject was required to estimate verbally the body tilt in degrees. After the estimation of body tilt, he or she was asked to open his or her eyes (binocular vision) and was required to adjust the luminous line to the apparent gravitational vertical from a randomly starting position at $20^{\circ}$ clockwise or counterclockwise from the true vertical. The subject pushed the button of the joystick when the adjustment was completed. Then the rod was switched off, the subject was told to close his or her eyes, and the chair was rotated to the next preset position.

The strapped and the body cast conditions were the objects of two separate sessions of approximately $45 \mathrm{~min}$ each. The order of sessions was counterbalanced over subjects. Each session included 30 trials, 15 angles of roll tilt repeated twice. The tilts of the chair were $0^{\circ}, \pm 15^{\circ}, \pm 30^{\circ}, \pm 45^{\circ}, \pm 60^{\circ}, \pm 75^{\circ}, \pm 90^{\circ}$, and $\pm 105^{\circ}$. The sequence of trials was randomly determined. The trials alternated left and right body tilts with respect to the gravitational vertical, to maintain the adaptation of the upright body to gravity (Higashiyama \& Koga, 1998). Another condition was that the subjects would never be placed in an upside down position when they were being moved between trials. Before each session, the subjects were showed a circle on which was drawn the $360^{\circ}$ by steps of $15^{\circ}$; they were told to imagine their head to be at the center of the circle and their body displaced at different angles of this scale. The subjects knew that the tilt values used were limited to 0,15 and its multiples, left and right. They received no feedback about their performances.

Data analyses were carried out on the angular difference, expressed in degrees, between the body or rod response and the posi- tion of the standard (physical tilt and upright, respectively). For the body-upright condition, the subjective vertical and body orientation errors were calculated using the algebraic mean settings. For the body tilt condition, rod adjustment errors were arbitrarily considered to be positive if they were in the direction of the body tilt (A-effect) and negative if they were in the opposite direction (E-effect). Body estimation errors were considered to be positive if the response was between the standard and $180^{\circ}$ (overestimation) and negative if the response was between the physical body tilt and the upright (underestimation).

Asymmetries described in the literature regarding the SVV assessment (Bauermeister, 1978) and the body tilt estimation (Pearson \& Hauty, 1959) are uncommon, and little is known about them. Two (side tilts, left vs. right) $\times 7$ (tilt orientations) analyses of variance (ANOVAs) with repeated measures on both factors were applied to the rod adjustment and to the body estimation errors. Since the results showed no difference between the right and the left tilts $(p>.05)$, errors for left and right tilts were pooled, and the algebraic mean settings of the four trials were calculated as the measure of the effect.

\section{RESULTS}

\section{Preliminary Experiment}

In order to investigate the alteration of gravity-based tactile stimulation created by the body cast, we restrained 2 subjects ( 1 male, $1.78 \mathrm{~m}, 72 \mathrm{~kg}$; 1 female, $1.64 \mathrm{~m}, 56$ $\mathrm{kg}$ ) on the movable platform in the two strapped and body cast conditions. The subjects were tilted at roll angles ranging from $0^{\circ}$ to $105^{\circ}$ by steps of $15^{\circ}$; only right tilts were tested. Pressure variations were recorded at six symmetrical parts on the left and right sides of the body by means of force-sensing resistance (FSR). The variation of resistance as a function of force gives an estimation of the variation of pressure generated at the level of the captor, with the output resistance increasing as the 

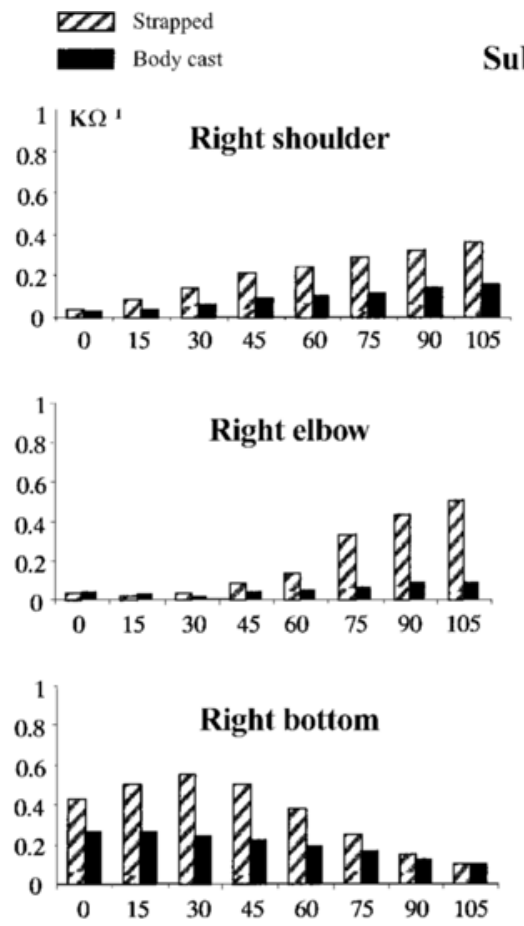

\section{Subject 1}
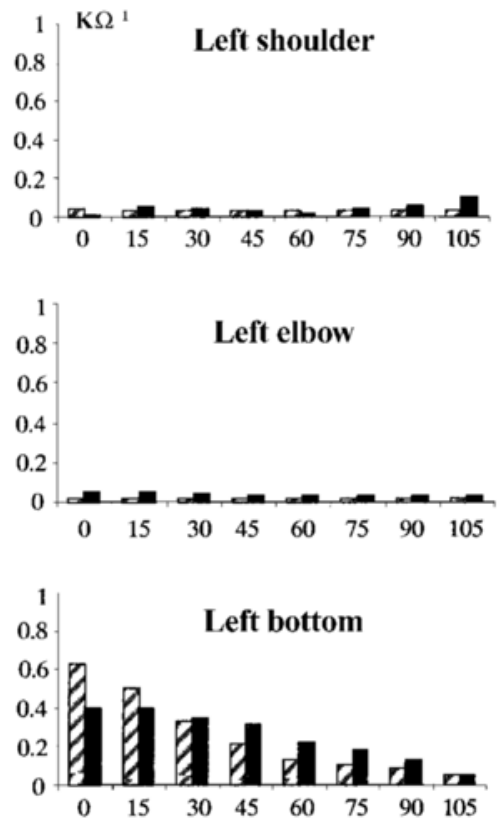

Subject 2
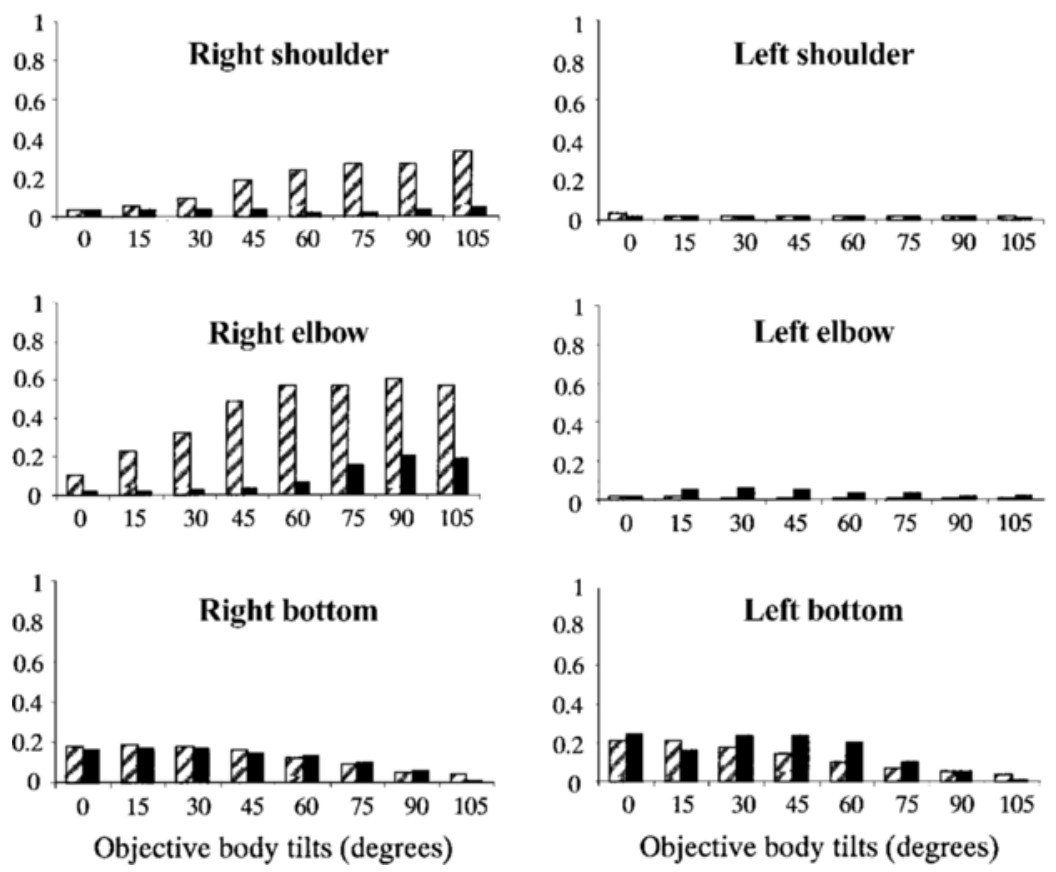

Figure 2. Evolution of pressure-1/resistance $(\mathrm{k} \Omega)$-for the six captors located at six body places as a function of objective body tilt (in degrees) for the two restriction conditions (control, striped plot; body cast, dark plot) and for the 2 subjects, one male (Subject 1) and one female (Subject 2).

force decreases. The six FSR locations were distributed as follows: one on each shoulder at the level of the deltoid proximal insertion, one on each elbow (epicondyl), and one on each ischiatic tuberosity.
As is illustrated in Figure 2, results obtained from the 2 subjects did not differ. This observation showed that the different morphological characteristics of the subjects did not interact with the distribution and evolution 
of pressure cues with respect to body orientation. We first examined the variations of pressure indirectly measured by the FSR obtained on the right side for the different right tilts. Concerning the shoulder and the elbow locations, results showed in the strapped, as in the body cast, condition that the pressure values increased linearly from the vertical to the $105^{\circ}$ tilt value. However, in the body cast condition, the pressure values for these two locations remained smaller than those in the strapped condition whatever the body tilt. Under the ischiatic tuberosity, pressure values decreased in both conditions. This suggested that the gluteal part of the body took off from the chair for larger tilts, leading progressively to no contact with the FSR. For each restrained condition, we also compared the difference of pressure indirectly measured by the FSR between right and left sides. In the strapped condition, results showed a greater pressure difference between the two FSRs placed at the symmetrical location, which increased with the roll tilt until $105^{\circ}$, whereas in the body cast the pressure difference remained smaller.

Thus, in the strapped condition, roll tilts induced a consistent modification in the repartition of pressure exerted on the body by the support surface. The extent of this modification depended on the importance of the tilt value. The pressure, generated by the antigravity reaction forces, increased on the contact zone with the increased tilt, and the pressure distribution became more asymmetrical when the tilt increased. In the body cast condition, however, the changing pressure pattern generated during body tilt was nearly invariant, whatever the body tilt. Consequently, as compared with the strapped condition, it can be stated that gravity-based somesthetic cues were greatly reduced in the body cast.

\section{Subjective Visual Vertical}

Preliminary data analyses were applied to the mean rod settings with the chair in an upright position, to detect the body restriction effect on SVV in the upright ori- entation. A one-way ANOVA showed that mean adjustment errors failed to differ between both body restriction conditions $[F(1,15)=0.72, p>.05]$. They also failed to differ significantly from zero $(p>.05)$. As has been indicated in the literature, we found that a strapped subject sitting in the vertical position in the dark can adjust the rod to the vertical with great accuracy. Furthermore, the body cast did not modify the SVV when the subject was upright.

A 7 (tilt orientations) $\times 2$ (body restriction conditions, strapped and body cast) ANOVA with repeated measures on the two factors was applied to the adjustment errors. Results revealed a significant effect of tilt orientation $[F(6,90)=56.62, p<.001]$ and of body restriction condition $[F(1,15)=31.38, p<.001]$ and a significant interaction of these factors $[F(6,90)=5, p<.001]$. Thus, the direction of errors depended on the tilt, with an A-effect at high tilt values. In the body cast condition, the SVV settings deviated more in the direction of body tilt than they did in the strapped condition (Figure 3). A post hoc analysis (Newman-Keuls, $p<.05$ ) showed that the difference between the two conditions was significant for the greater angle tilts only $\left(60^{\circ}, 75^{\circ}, 90^{\circ}\right.$, and $\left.105^{\circ}\right)$.

\section{Perceived Body Orientation}

A one-way ANOVA applied to the mean body estimation with the upright chair showed no significant effect of body restriction $[F(1,15)=1.69, p>.05]$. Moreover, estimation errors failed to differ significantly from zero $(p>.05)$. As has been reported in the literature, we found that a strapped subject sitting in the vertical position in the dark can establish that he or she is in the vertical position. Furthermore, the body cast did not modify this estimation of the vertical position.

A 7 (tilt orientations) $\times 2$ (body restriction conditions, strapped and body cast) ANOVA with repeated measures on the two factors was applied to the body estimation errors. Results showed a significant tilt orientation effect

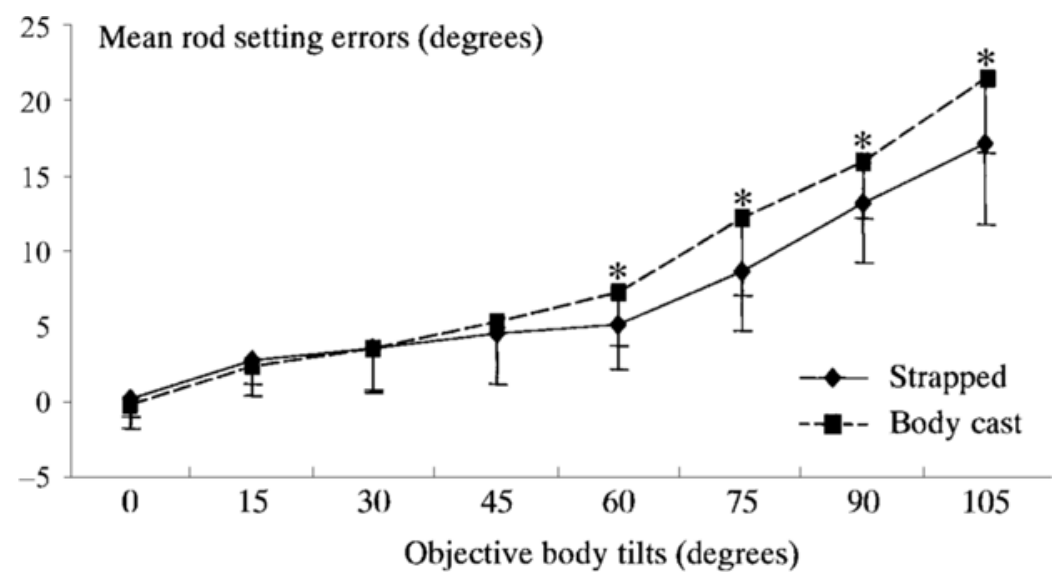

Figure 3. Mean rod setting errors (in degrees) for the strapped (solid line) and the body cast (dashed line) conditions as a function of roll tilts (in degrees). Error bars indicate standard deviations. $* p<.05$ denotes a significant difference between conditions. 
$[F(6,90)=6.34, p<.001]$ and no body restriction effect $[F(1,15)=11.22, p>.05]$, but a significant interaction of these factors $[F(6,90)=3.2, p<.005]$. A post hoc analysis (Newman-Keuls, $p<.05)$ confirmed two different trends (Figure 4). As compared with the strapped condition, the body tilt was underestimated with the body cast at $45^{\circ}$, and it was overestimated for body tilts values of $75^{\circ}$ and above.

Moreover, Figure 4 suggested that there were small or no estimation errors in the control condition. Indeed, when a least squares line was fitted to the mean tilts estimations in this condition, the equation was $y=0.99 x+$ 0.18 (95\% confidence interval), suggesting a correct estimation of the body orientation for all tilts. Conversely, in the body cast condition, a shallow slope for small tilts and a steep slope for large tilts were observed. Thus, two linear functions fitted to the mean estimation were calculated for small $\left(0^{\circ}-60^{\circ}\right)$ and large $\left(75^{\circ}-105^{\circ}\right)$ tilts separately. The mean line taken was $y=0.84 x+2.6$ for small tilts and $y=1.14 x-8.9$ for larger tilts, which confirm underestimation for small tilts and overestimation for large ones.

\section{Relationship Between the SVV and the Perceived Body Orientation}

A 2 (tasks, SVV and perceived body orientation) $\times 8$ (tilt orientation angles) $\times 2$ (body restriction, strapped and body cast) ANOVA with repeated measures on the three factors was applied to the estimation errors. Results showed significant effects of task $[F(1,15)=29.13$, $p<.001]$, body restriction condition $[F(1,15)=9.49, p<$ $.01]$, and tilt angle $[F(7,105)=38.54, p<.001]$. A post hoc analysis (Newman-Keuls, $p<.05)$ showed that, whatever the body restriction condition, SVV estimation errors were greater than the errors produced in assessing body orientation.

To further evaluate the relation between SVV adjustments and the perceived position, an analysis of correla- tion between body orientation errors and rod adjustment errors was made for each tilt angle. This was done for strapped and body cast restrictions separately. Results failed to reveal any significant correlation whatever the body restriction or body tilt condition (Bravais-Pearson, $p>.20$ ). A 8 (tilt orientations) $\times 2$ (body restriction conditions, strapped and body cast) analysis of covariation with repeated measures on the two factors was applied to the SVV adjustments, with body orientation estimations as the covariant factor. Results showed that the effects of the tilt angle $[F(7,98)=12.36, p<.01]$ and of the body restriction conditions $[F(1,14)=33.81, p<.01]$ remained significant. Thus, the body orientation estimation did not account for the SVV errors.

Another way of expressing the relation between both perceptual tasks is in terms of the apparent angle between the body orientation perception and the SVV for each body restriction condition. For each subject and each tilt, the apparent angle was the angular distance between the body orientation estimation and the SVV (perceived body orientation minus SVV adjustment). A 2 (body restriction, strapped and body cast) $\times 8$ (tilt orientation angles) ANOVA with repeated measures on the two factors was applied to the apparent angle. Results showed a significant effect of tilt angle $[F(7,105)=$ $359.17, p<.001]$, no effect of body restriction $[F(1,15)=$ $0.28, p>.5$ ] , and no interaction $(p>.46)$. As is shown in Figure 5, for each tilt, the same apparent angle was observed for the strapped and the body cast conditions. When compared with the objective angle (objective body tilt minus the gravitational gravity), the apparent angle was smaller for $30^{\circ}$ and above $(p<.05)$, and increasing body tilt induced a decrease of the apparent angle. Moreover, there was a relationship between the apparent angles in the strapped and the body cast conditions (BravaisPearson test, $r>.49, p<.05)$. Thus, for each individual and body tilt, there was a specific relation between the

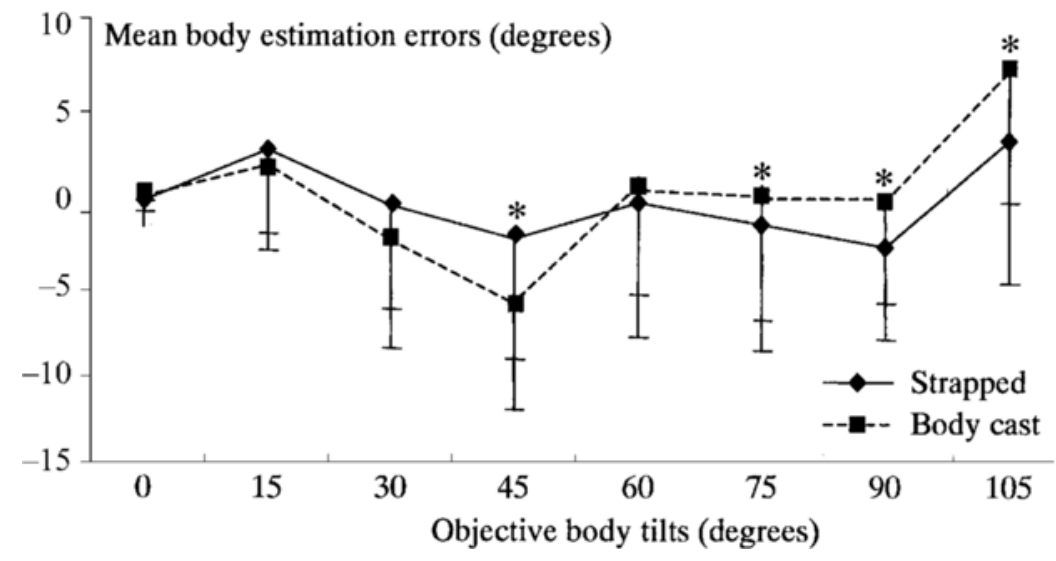

Figure 4. Mean estimation errors in the perceived body orientation (in degrees) for the strapped (solid line) and the body cast (dashed line) conditions as a function of roll tilts (in degrees). Error bars indicate standard deviations. $* p<.05$ denotes a significant difference between conditions. 


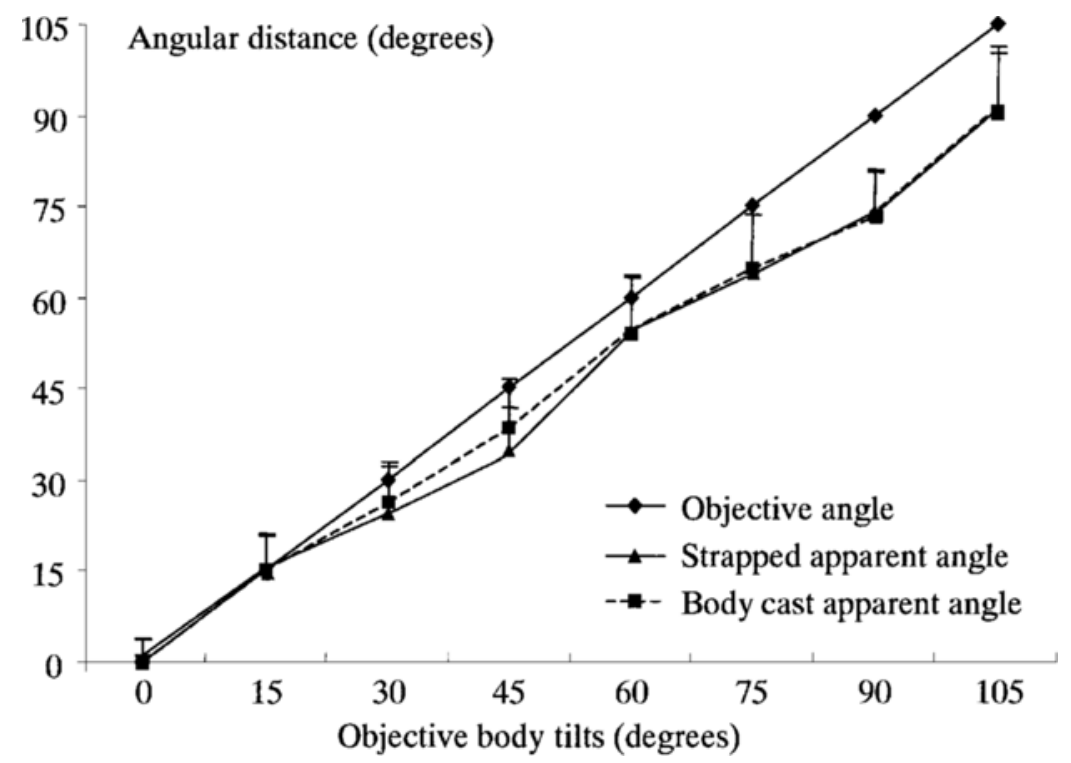

Figure 5. Angular distance between body orientation and the vertical: apparent angles (perceived body orientation minus SVV) for the strapped (triangles) and the body cast (squares) conditions and objective angle (objective body tilt minus the gravitational gravity; diamonds) as a function of roll tilts (in degrees). Error bars indicate standard deviations.

body and the external space, which did not change under the different restriction conditions but was shifted according to the available somesthetic cues.

\section{DISCUSSION}

This study focused on the role of the body orientation estimation on the SVV. In order to point out the link between those two perceptual tasks, we assumed that the somatosensory cues, which give information about gravity and then about the orientation of the body, could be used in the judgment of the SVV.

We first examined results in the control condition. Concerning the perceived body orientation, we found a general accuracy of the body tilt estimation that differs from that shown in the literature. Indeed, for large body tilts Higashiyama and Koga (1998) found an overestimation, whereas Van Beuzekom and Van Gisbergen (2000) observed a systematic underestimation whatever the body tilt. Some difference in the procedure could explain this inconsistency. The subjects were asked to estimate body tilt only with values of $15^{\circ}$ and its multiples. This method uses a less sensitive scale than does estimation across the entire range, and it may be easier to discriminate between $15^{\circ}$ step than $1^{\circ}$ step orientations. This choice may have led to improved body tilt estimates. On the other hand, in the SVV judgment, data showed systematic positive errors (A-effect), which became greater for the larger tilt (above $45^{\circ}$ ). The absence of the E-effect for the smaller tilts was not surprising; several authors have found an A-effect, instead of an E-effect, for those lateral tilts
(Wade, 1972; Witkin \& Asch, 1948). Thus, some subjects were resistant to the E-effect, whereas the A-effect exists systematically (Sandström, 1954; Udo de Haes, 1970).

In the body cast condition, with regard to body orientation perception, results showed a nonlinear modification of the estimation of the position of the body in space. An underestimation of the body tilt was observed at angle values below $60^{\circ}$, whereas at values larger than $60^{\circ}$ an overestimation of the body tilt was observed. With respect to the SVV in the body cast condition, the A-effect was more important, especially for the greater tilts $\left(\right.$ above $60^{\circ}$ ). Since the otolith organs produced the same gravity response in both conditions, these first results suggest that the somatosensory inputs are required for both body tilt and SVV estimations.

The somesthetic information available for the body cast induced smaller asymmetries and pressure intensities. We suggested that a more diffuse and isotropic pattern of pressure stimulation would lead to a body underestimation increase, whereas results showed an overestimation. However, in the body cast, the antigravity muscle tone was also reduced. Dietz, Gollhofer, Kleiber, and Trippel (1992) considered that the changes in muscular tension opposing the attraction of gravity may play a role in perceiving a body tilt. Teasdale et al. (1999) showed that accurate perception of body orientation was improved when proprioceptive information could be dynamically integrated. In line with this, Van Beuzekom, Medendorp, and Van Gisbergen (2001) found an improvement of the sense of self-tilt when subjects were active (somatosensory inputs resulting from muscular effort in postural 
control were available), as compared with a passive condition of body roll tilt. However, in the control situation of the present experiment, the subjects were restrained so that they were not free to oscillate about a given joint; they were in some way completely immobilized, as in the body cast condition. Therefore, one can assume that the results obtained in the body cast condition were caused by the changes in tactile mechanoreceptors stimulation distributed over the wide area of cutaneous tissue. Thus, as was suggested by Higashiyama and Koga (1998), the tactile mechanoreceptors provide the central nervous system with spatially and temporally coded information associated with changes in the distribution of pressure on skin that result from changes in body orientation.

The results do not provide us with a complete explanation for the larger systematic errors that took the form of an A-effect in the disturbed condition although the subjects tend to feel more tilted than they actually are. Conversely to our hypothesis, the body orientation estimation did not account for the SVV errors. Moreover, the absence of relationship between the two tasks suggest separate mechanisms for the SVV and body estimation under body tilt. However, the verbal estimation method used may be responsible too for the lack of correlation $\left(15^{\circ} \mathrm{vs} .0 .01^{\circ}\right.$ accuracy for body tilt and SVV estimations, respectively). Nevertheless, in the body cast condition, the same response shift occurred in the same direction for both the SVV and the perceived body orientation. It may be assumed that the change in somatosensory pattern inputs has a similar impact on the cognitive processes involved in assessing the perceptual tasks. One explanation of the body cast effect focuses on the process of integration of the available information. Van Beuzekom et al. (2001) proposed that an otolith signal, combined with nonvestibular gravity signals, such as somatosensory cues, provides an almost veridical representation of body in space, which serves as the basis for the self-estimates and is used in the SVV task. According to Mittelstaedt (1983), an internal representation of the body axis (z-axis) - that is, a virtual line running from the feet to the head-would be used as a reference frame for both the perception of body orientation and the SVV separately. The A-effect might be the result of the subject's tendency to shift the SVV toward this longitudinal body axis ( $z$-axis); this so-called idiotropic vector could be considered as a self-centered reference (Mittelstaedt, 1983). The $z$-axis would not be defined with respect to a specific sensory system. Rather, it would be a sensory integration in which the somesthetic contribution grows with tilt. The body cast condition is a particular environment in which somatosensory redundancy is limited: The patterns of pressure distribution and muscle tone are unusual, whereas interoception does not change. Interoception might play a greater role in building the self-centered reference, because of the interference produced by other somesthetic afferents (Mittelstaedt, 1997). The use of a body cast may result in modifying the spatial perception of one's own body through the weighting of sensory systems. As was suggested by von Holst
(1950), the relative importance, or weighting, of the information from the different receptors changes under a variety of conditions, including alterations of somatosensory cues. Moreover, Ito and Gresty (1997) suggested that the missing congruence between somatosensory cues raises the question that spatial disorientation per se may interfere with thinking processes involved in perceived body orientation and SVV. On the basis of the verbal reports of our subjects, there was indeed a subjective feeling of spatial disorientation in the body cast.

In conclusion, it can be argued that the somatosensory inputs, which convey information about the body-supportsurface interface, are required during body tilt to estimate the SVV and the body orientation. The relationship between the assessment of the spatial position of the body and the SVV remains unclear. The construction of an egocentric reference for somesthetic information, especially for greater subject tilt values, could account for a link between those two perceptual tasks. Further research on SVV and postural estimation with some spatial deficiencies, such as the spatial neglect syndrome, may prove valuable in better understanding the role of the egocentric reference in the orientation in space, as well as the somesthetic contribution to the self-centered reference.

\section{REFERENCES}

Anastasopoulos, D., Bronstein, A., Haslwanter, T., Fetter, M., \& Dichgans, J. (1999). The role of somatosensory input for the perception of verticality. In B. Cohen \& B. J. M. Hess (Eds.), Otolith function in spatial orientation and movement (Annals of the New York Academy of Sciences, Vol. 871, pp. 379-383). New York: New York Academy of Sciences.

BAuermeister, M. (1978). Differences between right versus left lateral body tilt in its effect on the visual and tactual perception. Psychological Research, 40, 183-187.

Bringoux, L., Nougier, V., Barraud, P. A., Marin, L., \& Raphel, C. (in press). Contribution of somaesthetic information to the perception of body orientation in the pitch dimension. Quarterly Journal of Experimental Psychology.

Bronstein, A. M. (1999). The interaction of otolith and proprioceptive information in the perception of verticality: The effects of labyrinthine and CNS disease. In B. Cohen \& B. J. M. Hess (Eds.), Otolith function in spatial orientation and movement (Annals of the New York Academy of Sciences, Vol. 871, pp. 324-333). New York: New York Academy of Sciences.

Brown, J. L. (1961). Orientation to the vertical during water immersion. Aerospace Medicine, 32, 209-217.

DAY, R. H., \& WADE, N. J. (1969). Mechanisms involved in visual orientation constancy. Psychological Bulletin, 71, 33-42.

Dietz, V., Gollhofer, A., Kleiber, M., \& Trippel, M. (1992). Regulation of bipedal stance: Dependence on "load" receptors. Experimental Brain Research, 89, 229-231.

Higashiy ama, A., \& Koga, K. (1998). Apparent body tilt and postural aftereffect. Perception \& Psychophysics, 60, 331-347.

HowARD, I. P. (1986). The perception of posture, self motion, and the visual vertical. In K. R. Boff, L. Kaufmann, \& J. P. Thomas (Eds.), Handbook of perception and human performance (Vol. 1, 18-1 to 18-62). New York: Wiley.

Howard, I. P., \& Templeton, W. B. (1966). Human spatial orientation. New York: Wiley.

Ito, Y., \& GRESTY, M. A. (1997). Subjective postural orientation and visual vertical during slow pitch tilt for the seated human subject. Aviation, Space, \& Environmental Medicine, 68, 3-12.

JARCHOW, T., \& MAST, F. W. (1999). The effect of water immersion on 
postural and visual orientation. Aviation, Space, \& Environmental Medicine, 70, 879-886.

LECHNER-STEINLEITNER,S., \& SCHÖNE, H. (1980). The subjective vertical under "dry" and "wet" conditions at clockwise and counterclockwise changed positions and the effect of a parallel background field. Psychological Research, 41, 305-317.

MAST, F. W., \& JARCHOW, T. (1996). Perceived body position and the visual horizontal. Brain Research Bulletin, 40, 393-397.

Mittelstaedt, H. (1983). A new solution to the problem of the subjective vertical. Naturwissenschaften, 70, 272-281.

MittelstaedT, H. (1992). Somatic versus vestibular gravity reception in man. In B. Cohen, D. L. Tomko, \& F. E. Guedry (Eds.), Sensing and controlling motion, vestibular and sensorimotor function (Annals of the New York Academy of Sciences, Vol. 656, pp. 124-139). New York: New York Academy of Sciences.

Mittelstaedt, H. (1997). Interaction of eye-, head-, and trunk-bound information in spatial perception and control. Journal of Vestibular Research, 7, 283-302.

Nelson, J. G. (1968). Effect of water immersion and body position upon position of gravitational vertical. Aerospace Medicine, 39, 806811.

Nemire, K., \& Cohen, M. M. (1993). Visual and somesthetic influences on postural orientation in the median plane. Perception \& Psychophysics, 53, 106-116.

Ny воRG, H. (1971). Tactile stimulation and perception of the vertical: I. Effect of diffuse vs. specific tactile stimulation. Scandinavian Journal of Psychology, 12, 1-13.

Parker, D. E., Poston, R. L., \& Gulledge, W. L. (1983). Spatial orientation: Visual-vestibular-somatic interaction. Perception \& Psychophysics, 33, 139-146.

Pearson, R. G., \& Hauty, G. T. (1959). Adaptive processes determining proprioceptive perception of verticality. Journal of Experimental Psychology, 57, 367-371.

RAPHAN, T., \& STURM, D. (1992). Modeling the spatiotemporal organization of velocity storage in the vestibuloocular reflex by optokinetic studies. Journal of Neurophysiology, 66, 1410-1421.

SANDST RÖM, C. I. (1954). A note on the Aubert phenomenon. Journal of Experimental Psychology, 48, 209-210.
SCHÖNE, H. (1964). On the role of gravity in human spatial orientation. Aerospace Medicine, 35, 764-772.

SchöNE, H. (1975). The "weight" of the gravity organ's signal in the control of perceptual and reflex type orientation at different body positions. Fortschritte der Zoologie, 23, 274-285.

SchÖNE, H., \& WADE, N. J. (1971). The influence of force magnitude on the perception of body position. British Journal of Psychology, 62, 347-352.

Teasdale, N., Nougier, V., Barraud, P. A., Bourdin, C., Debû, B., Poquin, D., \& RAPHEL, C. (1999). Contribution of ankle, knee, and hip joints to the perception threshold for support surface rotation. Perception \& Psychophysics, 61, 615-624.

Udo de Haes, H. A. (1970). Stability of apparent vertical and ocular countertorsion as a function of lateral tilt. Perception \& Psychophysics, 8, 137-142.

Van Beuzekom, A. D., Medendorp, W. P., \& Van Gisbergen, J. A. M. (2001). The subjective vertical and the sense of self orientation during active body tilt. Vision Research, 41, 3229-3242.

Van Beuzenom, A. D., \& Van Gisbergen, J. A. M. (2000). Properties of the internal representation of gravity inferred from spatial-direction and body-tilt estimates. Journal of Neurophysiology, 84, 11-27.

von Holst, E. (1950). Die Arbeitsweise des Statolithenapparates bei Fischen. Zeitschrift für vergleichende Physiologie, 32, 60-120.

WADE, N. J. (1972). Effect of forward head inclination on visual orientation during lateral body tilt. Journal of Experimental Psychology, 96, 203-205.

WADE, N. J. (1973). The effect of water immersion on the perception of the visual vertical. British Journal of Psychology, 64, 351-361.

Wit KIN, H. A., \& Asch, S. E. (1948). Studies in space orientation: III. Perception of the upright in the absence of a visual field. Journal of Experimental Psychology, 38, 603-614.

YARDLEY, L. (1990). Contribution of somatosensory information to perception of the visual vertical with body tilt and rotating visual field. Perception \& Psychophysics, 48, 131-134.

(Manuscript received May 8, 2002;

revision accepted for publication April 4, 2003.) 